\title{
The non-specific lipid transfer protein from hazelnut, Cor a 8, a relevant food allergen
}

\author{
Pawel Dubiela ${ }^{1 *}$, Sabine Pfeifer ${ }^{1}$, Merima Bublin ${ }^{1,2}$, Cristine Hafner ${ }^{1}$, Karin Hoffmann-Sommergruber ${ }^{1}$ \\ From Food Allergy and Anaphylaxis Meeting 2014 \\ Dublin, Ireland. 9-11 October 2014
}

\section{Background and aim}

Hazelnuts are potent inducers of food allergic symptoms, ranging from mild up to severe reactions in sensitized individuals. So far, 10 hazelnut allergens were identified, among those the ns lipid transfer protein, Cor a 8 . The aim of the current study is to purify and characterise the physicochemical and allergenic properties of Cor a 8.

\section{Methods}

nCor a 8 was purified from raw hazelnuts by precipitation and chromatographical steps. Subsequently, the protein was identified by $\mathrm{N}$-terminal sequencing and mass spectrometry. Stability of the protein was investigated by simulated gastric and duodenal digestion assays. Hazelnut lipids were extracted employing the hexane method. Finally, IgE binding activity of native and reduced/alkylated nCor a 8 was tested in ELISA using sera from 6 hazelnut allergic patients.

\section{Results}

Purified nCor a 8 migrates in SDS PAGE as a single band at around $12 \mathrm{kDa}$. MALDI TOF mass spectrometry provided $9.475 \mathrm{kDa}$ corresponding to the theoretical mass of $9.468 \mathrm{kDa}$ (database access. number: 9QATH2). The intact $\mathrm{n}$-terminus was verified by Edman-degradation verifying the first 6 amino acid residues. In digestion assays purified Cor a 8 displayed high stability against enzymatic treatment.

IgE binding activity was tested in sera from 6 hazelnut allergic patients. Three out of those sera had IgE predominantly recognizing linear epitopes as compared to the other 3 sera which had IgE specific for conformational epitopes. In RBL assays Cor a 8 induced mediator release in a dose dependent manner. Upon addition of hazelnut lipids this response was remarkably increased.

\section{Conclusions}

Cor a 8 was purified from raw hazelnut extract, displaying the physicochemical properties of a member of the nsLTP protein family, such as high resistance against enzymatic degradation. In cellular assays the allergenic activity of Cor a 8 was considerably increased when adding hazelnut lipids, thus showing the impact of food matrix on the allergenicity of a single food protein.

\section{Acknowledgements}

Supported by grants SFB F4603 and W1248 (Austrian Science Fund) to K. Hoffmann-Sommergruber and P. Dubiela, respectively.

\section{Authors' details}

${ }^{1}$ Department of Pathophysiology and Allergy Research, Medical University of Vienna, Vienna, Austria. ${ }^{2}$ Karl Landsteiner Institute for Dermatological

Research, St. Poelten, Austria.

Published: 30 March 2015 